\title{
Niedrigere Werte sind gut erreichbar
}

Fragestellung: Lässt sich eine aggressivere antihypertensive Therapie bei Patienten nach transienter ischämischer Attacke (TIA) und Schlaganfall in der klinischen Praxis erreichen?

Hintergrund: Die arterielle Hypertonie ist der häufigste und wichtigste Risikofaktor für den Schlaganfall. Daher ist eine konsequente antihypertensive Therapie von eminenter Bedeutung. Die Risikoreduktion bei der Senkung des hohen Blutdrucks ist für zerebrale Blutungen höher und wichtiger als für zerebrale Ischämien. Zurzeit empfehlen Leitlinien für Patienten ohne Diabetes mellitus den systolischen Blutdruck unter $140 \mathrm{~mm} \mathrm{Hg}$ zu senken. Bei Patienten mit Diabetes mellitus wird eine Grenze von $130 \mathrm{~mm} \mathrm{Hg}$ empfohlen. In den Leitlinien zur Sekundärprävention des Schlaganfalls finden sich international beide Grenzen. Eine offene Fragestellung ist, wie gut es gelingt, bei Patienten in der klinischen Praxis die entsprechenden Grenzwerte für den systolischen Blutdruck therapeutisch zu erreichen.

Patienten und Methodik: Die Studie wurde in 99 Praxen von niedergelassenen praktischen Ärzten in England im Zeitraum zwischen 2009 und 2011 durchgeführt. Alle Patienten hatten einen Schlaganfall oder eine TIA erlitten und der systolische Blutdruck lag über $125 \mathrm{~mm} \mathrm{Hg}$. Die Patienten wurden in zwei Therapiegruppen randomisiert. Es sollte entweder ein Grenzblutdruck von $130 \mathrm{~mm} \mathrm{Hg}$ oder von $140 \mathrm{~mm} \mathrm{Hg}$ erreicht werden. Die Behandlung selbst war den Hausärzten überlassen. Die Blutdruckwerte wurden über zwölf Monate erfasst, wobei

Mant J, McManus RJ, Roalfe A et al. Different systolic blood pressure targets for people with history of stroke or transient ischaemic attack: PAST-BP (Prevention After Stroke-Blood Pressure) randomised controlled trial. BMJ 2016; 352: i708 der Endpunkt, der systolische Blutdruck, nach zwölf Monaten gemessen wurde.

Ergebnisse: In die Studie wurden 529 Patienten mit einem mittleren Alter von 63 Jahren eingeschlossen. 266

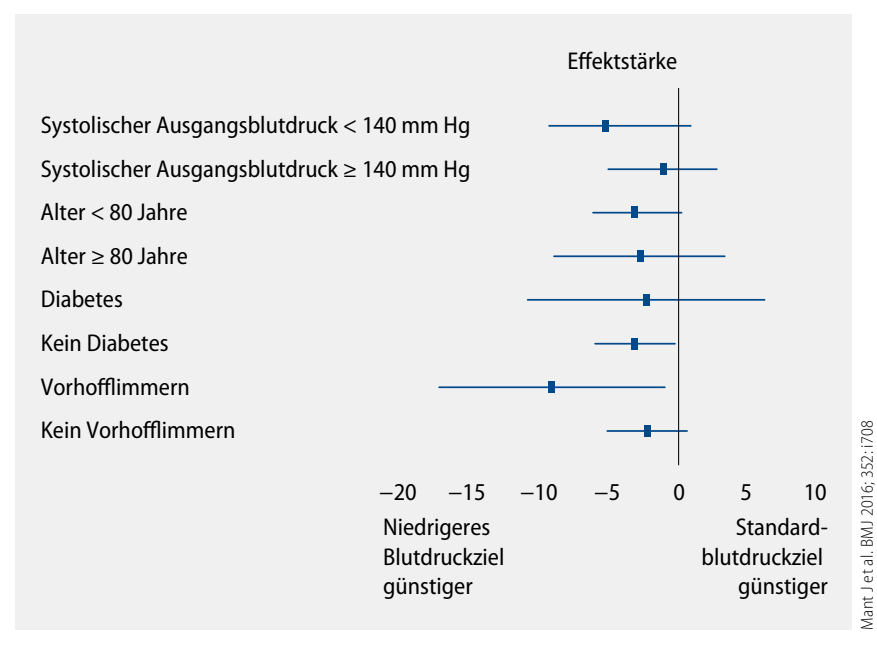

1 Effekte von niedrigerem versus Standardblutdruckziel.

der Patienten sollten einen systolischen Blutdruckwert $<130$ mm Hg und 263 einen systolischen Blutdruck $<140$ mm Hg erreichen. Für die Endauswertung standen die Ergebnisse von 379 Patienten zur Verfügung. Nach zwölf Monaten fiel der systolische Blutdruck in der intensiven Therapiegruppe um $16,1 \mathrm{~mm} \mathrm{Hg}$ auf $127,4 \mathrm{~mm} \mathrm{Hg}$. In der Standardtherapiegruppe fiel der Blutdruck um 12,8 mm Hg auf einen Mittelwert von $129 \mathrm{~mm} \mathrm{Hg}$. Dieser Unterschied war statistisch signifikant. Die Unterschiede waren am ausgeprägtesten bei Patienten, die zusätzlich unter Vorhofflimmern litten. Bei einer systematischen Abfrage von potenziellen Nebenwirkungen zeigen sich keinerlei Unterschiede zwischen den beiden Behandlungsregimen.

Schlussfolgerungen: Im klinischen Alltag ist es möglich, bei Patienten nach TIA und Schlaganfall und arterieller Hypertonie, systolische Blutdruckwerte von unter $130 \mathrm{~mm} \mathrm{Hg}$ zu erreichen.

\section{- Kommentar von Hans-Christoph Diener, Essen}

\section{Das niedrigere Blutdruckziel ist wahrscheinlich besser präventiv wirksam}

Randomisierte Therapiestudien, die Endpunkte untersuchen, sind wichtig und werden in aller Regel verblindet durchgeführt. Eine häufig ungelöste Frage ist anschließend, wie gut die Ergebnisse dieser Studie in den klinischen Alltag umgesetzt werden können. Die hier vorliegende Studie, die in den Praxen von niedergelassenen Hausärzten in England durchgeführt wurde, zeigt, dass es in dieser Patientenpopulation sehr gut möglich ist, ohne schwerwiegende Nebenwirkungen systolische Blutdruckwerte von $130 \mathrm{~mm} \mathrm{Hg}$ oder weniger zu erreichen. Sehr wahrscheinlich ist dieses Therapieziel präventiv besser wirksam als eine systolische Blutdruckgrenze von $140 \mathrm{~mm} \mathrm{Hg}$.

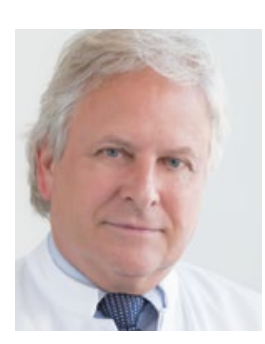

Prof. Dr. med. Hans-Christoph Diener, Essen

Seniorprofessur für klinische

Neurowissenschaften,

Universitätsklinikum Essen

E-Mail:h.diener@uni-essen.de 